\title{
A propos du Stenasel/us laticarpus Birstein (Crustacea Isopoda Asellota)
}

\author{
Guy MAGNIEZ*
}

Dans ce même fascicule de l'International Journal of Speleology est publié, à titre posthume, un travail rédigé il y a plusieurs années par le regretté Professeur J.A. Birstein, de l'Université de Moscou. La maladie, puis la disparition du grand carcinologiste russe sont à l'origine de ce retard considérable. Il s'agit de la description d'une espèce nouvelle de la famille des Stenasellidae (Asellota anophtalmes et apigmentés) provenant des nappes phréatiques d'Afrique occidentale (bassin du Niger).

Les Stenasellidae Dudich 1924 ont d'abord été connus par des espèces vivant dans les eaux karstiques, puis interstitielles de l'Europe méridionale. Toutes ces formes ont été rangées dans un unique genre (Stenasellus Dollfus 1897), dont l'espèce-type est le Stenasellus virei du Gouffre de Padirac (Lot, France). Or, à partir de 1938, des espèces extra-européennes ont été découvertes dans les eaux souterraines d'Afrique occidentale et équatoriale. L'habitude aidant, elles furent également classées dans le genre Stenasellus européen. En 1966,j'avais eu l'occassion de réexaminer la plupart des espèces africaines décrites.

J'avais alors conclu que les Stenasellidae africains, rangés dans le genre Stenasellus s.l. ne répondaient que fort mal à la diagnose générique de Stenasellus amendée par Racovitza en 1924 (Bull. Soc. Sc. Cluj, 2,84) et qu'il convenait d'y voir plusieurs genres indépendants (correspondant à autant de lignées évolutives naturelles, chacune occupant une aire géographique particulière), présentant des caractères morphologiques très différents de ceux de Stenasellus car. emend. Ainsi furent créés les genres Metastenasellus (Int. J. Speleol., 2, 179, 1966) pour les trois espèces du bassin hy drographique du Congo: M. leleupi (Chappuis), M. congolensis,(Chappuis), et $M$. dartevellei (Chappuis), et Parastenasellus (Int. J. Speleol. 2, 182, 1966), pour l'unique espèce très primitive $P$. chappuisi (Remy) dont les affinités avec les Parastenetrioidea marins sont évidentes. Quelques mois plus tard, le Professeur Lanza de Florence proposa de créer pour les "Stenasellus" restants d'Afrique occidentale (St. africanus Monod et St. guineensis Braga), le genre nouveau Magniezia (Monit. Zool. Ital., 74, 238, 1966), dont il donna une diagnose détaillée. Le bien fondé de cette manière moderne de concevoir le groupe des Stenasellidae, déjà entrevu par Dudich (1924) et Arcangeli (1938) a été constamment confirmé par la suite, par une série de découvertes dont le Professeur Birstein ne pût, malheureusement avoir connaissance. Les principales sont les suivantes:

- La capture, dans des sources du Sénégal d'une petite espèce que Sket a pu inclure d'emblée dans le genre Magniezia Lanza (M. studiosorum Sket, 1969), montrant ainsi $a$ posteriori la validité des observations de Lanza.

* Laboratoire de Biologie Animale et Générale, Faculté des Sciences de la Vie et de l'Environnement, 6 Bd. Gabriel, 21 Dijon, France. 
- La découverte en Afrique orientale, par Lanza et ses collaborateurs, d'une nouvelle espèce appartenant au genre Stenasellus.ss., prouvant que ce genre épuré forme maintenant une entité systématique et biogéographique plus homogène qu'antérieurement.

- La découverte, par deux zoologistes américains (Cole et Minckley 1970), de représentants de la famille des Stenasellidae dans les eaux souterraines d'Amérique centrale, apportant de nouvelles preuves que les Sténaselles ne sont pas un simple genre d'Asellidae, comme on avait tendance à le penser, mais un véritable groupe d'Asellotes anophtalmes, ayant évolué indépendamment du précédent, dans d'autres provinces biogéographiques. Ce groupe doit être considéré comme très ancien. Il est très isolé dans la faune des Asellotes actuels (Henry et Magniez, 1970, Magniez et Henry, 1970). Il est formé de plusieurs lignées isolées les unes des autres depuis longtemps, chacune ayant subi sa propre spéciation. Les genres africains Metastenasellus, Parastenasellus et Magniezia correspondent à certaines de ces lignées, qui ont évolué dans les eaux souterraines des boucliers nigérien et congolais et on doit les considérer comme des groupes naturels d'espèces parentes, au sein de la famille, c'est-à-dire des genres véritables. Le Stenasellus laticarpus décrit par Birstein, par les caractères de ses différents appendices: maxillules et pléopodes I, II, III, IV et V en particulier, se montre étroitement apparenté aux trois espèces d'Afrique occidentale: Magniezia africana (Monod), M. guineensis (Braga) et M. studiosorum Sket. Je propose donc de le considérer comme appartenant au genre Magniezia Lanza, sous le nom de Magniezia laticarpa (Birstein). A la suite de la très intéressante étude qu'il a faite de cette nouvelle espèce, il convient de souligner encore l'éminente contribution du Professeur J.A. Birstein à la connaissance de ce groupe d'Asellotes souterrains anophtalmes et apigmentés, dont il n'a malheureusement pu suivre les derniers développements de l'étude faunistique.

(terminé le 12 juillet 1971)

\section{R E S U M E}

En raison de sa parenté évidente avec trois autres espèces de Stenasellidae d'Afrique occidentale, rangées dans le genre Magniezia Lanza, Stenasellus laticarpus Birstein peut-être considéré comme appartenant à ce genre:

Stenasellus laticarpus $=$ Magniezia laticarpa $($ Birstein $)$.

\section{S U M M A R Y}

The species Stenasellus laticarpus Birstein is closely related to three west-african Stenasellidae, forming the genus Magniezia Lanza. It belongs to the same genus:

Stenasellus laticarpus $=$ Magniezia laticarpa $($ Birstein $)$. 


\section{B I B L I O G R A P H I E}

BIRSTEIN, J.A. 1951. Faune de 1'URSS. VII/5, Asellota. Moscou, 1-142.

BIRSTEIN, J.A. 1972. Une nouvelle espèce africaine du genre Stenasellus (Crustacea Isopoda Asellota) du bassin du Niger. Int. J. Speleol. 4, 9-18.

BIRSTEIN, J.A. et STAROSTIN, I.V. 1949. Sur une forme nouvelle pour l'URSS de Stenasellus provenant du Turkménistan (en russe). C.R. Acad. Sc. Moscou; N.S., 69, 691-694.

BRAGA, J.M. 1950. Sur deux Stenasellus (Crust. Isop.) de la Guinée portugaise. Ann. Fac. Sc. Porto, 35, 50-56.

COLE, G.A. and MINCKLEY, W.L. 1970. Sphaerolana, a new genus of Cirolanid Isopod from northern Mexico, with description of two new species. Southwestern Naturalist, USA, 15, 1, 71-81.

COLE, G.A. and MINCKLEY, W.L. (1971). Stenasellid Isopod Crustaceans in the Western Hemisphere - A new genus and species from Mexico - with a rewiew of other north american freshwater Isopod genera. Proc. Biol. Soc. Washington, (sous presse).

LANZA, B., CHELAZZI, L. et MESSANA, G. 1970. Stenasellus costai sp. n., Isopode freatobio gigante della Somalia. Monit. Zool. Ital., 5, 133-158.

MAGNIEZ, G. et HENRY, J.-P. 1970. Sur la structure des pléopodes copulateurs des Aselloidea (Crustacea Isopoda Asellota). C.R. Acad. Sc. Paris, 270, 93-95.

MONOD, Th. 1945. Un nouveau Stenasellus ouest-africain. Bull. I.F.A.N., 7, 101-114.

SKET, B. 1969. Eine neue Art der Stenasellinae (Isopoda Asellota) aus Senegal. Bull. Sc. Biol. Inst. Univ. Ljubljana, A, 14, 11/12, 386-387. 